\title{
Non-Orthogonal Multiple Access in Multiuser MIMO Configurations via Code Reuse and Principal Component Analysis
}

\author{
Panagiotis K. Gkonis* ${ }^{\mathbb{D}}$, Panagiotis T. Trakadas ${ }^{(D)}$ and Lambros E. Sarakis \\ General Department, National and Kapodistrian University of Athens, Sterea Ellada, \\ 34400 Dirfies Messapies, Greece; ptrakadas@uoa.gr (P.T.T.); lsarakis@uoa.gr (L.E.S.) \\ * Correspondence: pgkonis@uoa.gr
}

Received: 20 July 2020; Accepted: 13 August 2020; Published: 18 August 2020

check for updates

\begin{abstract}
The goal of the study presented in this paper is to evaluate the performance of a proposed transmission scheme in multiuser multiple-input multiple-output (MIMO) configurations, via code reuse. Hence, non-orthogonal multiple access (NOMA) is performed. To this end, a correlation matrix of the received data is constructed at the transmitter, with feedback as only the primary eigenvector of the equivalent channel matrix, which is derived after principal component analysis (PCA) at the receiver. Afterwards, users experiencing improved channel quality (i.e., diagonal terms of the correlation matrix) along with reduced multiple access interference (i.e., the inner product of transmission vectors) are the potential candidates for their assigned code to be reused. As the results indicate, considering various MIMO configurations, the proposed approach can achieve almost 33\% code assignment gain (CAG), when successive interference cancellation (SIC) is employed in mobile receivers. However, even in the absence of SIC, CAG is still maintained with a tolerable average bit error rate (BER) degradation.
\end{abstract}

Keywords: NOMA; PCA; MIMO systems

\section{Introduction}

The deployment of fifth-generation (5G) mobile cellular networks is inextricably connected with the provision of high data rates to mobile stations (MSs) in order support bandwidth demand and zero latency applications [1-3]. To this end, various novel technologies have been introduced over the last few years: mmWave transmission [4,5], massive multiple-input multiple-output (MIMO) systems [6,7], as well as non-orthogonal multiple access (NOMA) transmission schemes [8,9]. In the latter case, available network resources (i.e., frequency blocks or codewords) can be reused by certain groups of users, thus improving overall network capacity. Therefore, two main NOMA categories can be identified in literature: power domain NOMA [10,11] and code-domain NOMA [12]. In the first approach, the transmitted signals share the same frequency and time resources but vary in power. The second approach includes, among others, multiple access solutions relying on low-density spreading (LDS) and sparse code multiple access (SCMA).

In power domain NOMA, major scientific challenges include, among others, appropriate power allocation to MSs, and advanced transceiver techniques to mitigate intracode interference. In this context, MSs with improved channel conditions employ successive interference cancellation (SIC) to remove the messages intended for other MSs before decoding their own. In [13], the impact of user pairing on the performance of two NOMA systems, NOMA with fixed power allocation (F-NOMA) and cognitive radio inspired NOMA (CR-NOMA), was studied. According to the presented results, the performance gain of F-NOMA over conventional orthogonal multiple access (OMA) assignment schemes can be further improved by selecting users whose channel conditions are more distinctive. 
In [14], system-level evaluations were performed, according to which downlink NOMA with SIC can improve both the capacity and cell-edge user throughput performance based on wideband channel quality indicator (CQI) without relying on the availability of the frequency-selective CQI at the base station (BS) transmitter side. In this context, multi-user transmit power allocation and multi-user scheduling are performed according to sub-optimum algorithms. In [15], the design and performance evaluation of precoding and detection matrices for MIMO-NOMA is described. In this framework, user pairing was considered. However, users were randomly divided into multiple groups. The work in [16] focused on leveraging the capabilities provided by low-complexity single-antenna cooperative relays with buffers and NOMA transmission. In the same context, in [17], a novel relay selection policy is presented, namely flex-NOMA, that facilitates simultaneous transmissions from multiple sources to multiple relays, exploiting channel state information at the reception, and dynamic decoding ordering by the relays performing SIC. The work in [18] presented a sub-optimal hierarchical power allocation scheme along with a vertical user-pairing approach to deal with a large number of users in the NOMA system. An optimization problem is formulated to maximize the sum rate with total power and minimum rate constraints. In [19], the authors studied user pairing and power allocation in a downlink 2-user NOMA network. In addition, the globally-optimal closed-form solution in a general NOMA system was obtained. In [20], the paper focused on the user pairing and power allocation in the 2-user NOMA system and proposed the user pair power allocation (UPPA) scheme based on the optimal closed-form solution with the proportional fairness objective. Simulation results verified that the proposed UPPA scheme improves the performance gains of NOMA over orthogonal frequency division multiple access (OFDMA) in terms of both efficiency and user fairness.

It becomes apparent from the above that a major scientific challenge in power-domain NOMA schemes is the deployment of computationally efficient approaches for user pairing during transmission. In this work, a code reusage scheme is proposed and evaluated which separates active users into groups according to their channel quality and interuser interference. To this end, the received data correlation matrix is constructed at the transmitter, using only the primary eigenvector and eigenvalue of the corresponding correlation matrix as the input via feedback, deducted via principal component analysis (PCA) at MSs [21]. Hence, as it is shown in the presented results, our approach can achieve significant code assignment gain (CAG) with minimum computational and feedback complexity as well as average bit error rate (BER) deterioration. CAG is defined as the ratio of total codes assigned in the NOMA case compared to the ones used in the OMA transmission schemes.

The rest of this article is organized as follows: In Section 2, basic transceiver concepts of multiuser MIMO configurations are described. In Section 3, the proposed NOMA approach based on PCA and SIC is described. Simulation results are presented in Section 4, both for the SIC/non-SIC cases at MSs for various MIMO configurations. Finally, concluding remarks and proposals for future work are provided in Section 5 .

\section{Multiuser MIMO Configurations}

Considering the downlink of a MIMO configuration with $M_{t}$ antennas at the transmitter and $M_{r}$ antennas per MS ( $M_{t} \times M_{r}$ configuration), the transmitted signal can be expressed as [22]

$$
\mathbf{x}_{k}(t)=\sqrt{p_{k}} \mathbf{t}_{k} b_{k}(t) c_{k}(t)
$$

where $\mathbf{x}_{k}(t)$ is the $M_{t} \times 1$ transmission signal matrix of the $k$ th MS $(1 \leq k \leq K), p_{k}$ is the allocated power, $\mathbf{t}_{k}$ represents the $M_{t} \times 1$ precoding matrix, $b_{k}(t)$ is the transmitted symbol, while $c_{k}(t)$ is a spreading sequence for Code Division Multiple Access (CDMA) schemes [23]. Hence, in this context, a code-domain NOMA system was considered that can support multiple transmissions within the same time-frequency resource block by assigning different codes to different MSs [24]. 
The received signal at the sth symbol period (i.e., $[(s-1) \times T, s \times T]$ where $T$ is the symbol duration, for simplicity time indexes have been omitted) after Maximal Ratio Combining (MRC) via 2-D space-time RAKE processing is obtained by [25]

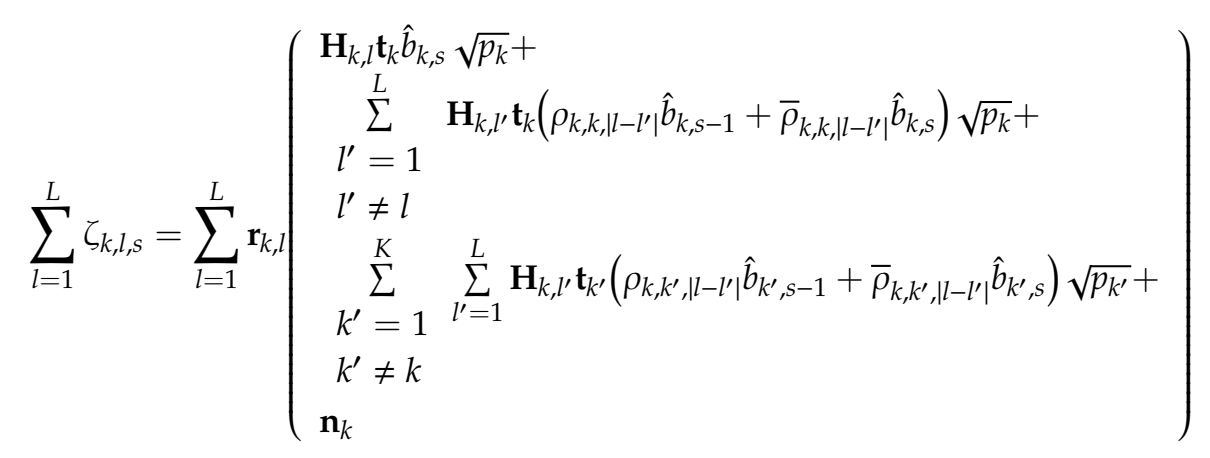

where the first term is the desired MS signal, the second/third term denotes multiple access and intersymbol interference, respectively, while the final term is the additive white Gaussian noise.

Moreover, $L$ is the number of multipath components, $\mathbf{r}_{k, l}=\left(\mathbf{H}_{k, l} \mathbf{t}_{k}\right)^{\mathrm{H}}$ is the MRC multiplying vector $\left(1 \leq l \leq L, \mathrm{x}^{\mathrm{H}}\right.$ is the conjugate transpose of $\left.\mathrm{x}\right), \mathbf{H}_{k, l}$ is the $M_{r} \times M_{t}$ channel matrix, $\hat{b}_{k, s}$ is the received symbol and $\rho, \bar{\rho}$ are the corresponding partial cross-correlations [21]. Hence, in a multiuser (MU) MIMO configuration, the equivalent signal to interference ratio (SIR) can be expressed as (neglecting intersymbol interference) [25]

$$
S I R_{k} \approx \frac{\left(\sum_{l=1}^{L} \sum_{q=1}^{M_{r}}\left|\sum_{v=1}^{M_{t}} \mathbf{H}_{k, l}^{(q, v)} \mathbf{t}_{k}^{(v)}\right|^{2}\right)^{2} p_{k}}{\sum_{\substack{k^{\prime}=1, k^{\prime} \neq k}}^{K} p_{k^{\prime}}\left|\sum_{l=1}^{L} \sum_{q=1}^{M_{r}}\left(\overline{\sum_{v=1}^{M_{t}} \mathbf{H}_{k, l}^{(q, v)} \mathbf{t}_{k}^{(v)}}\right) \sum_{l^{\prime}=1}^{L}\left(\sum_{v=1}^{M_{t}} \mathbf{H}_{k, l^{\prime}}^{(q, v)} \mathbf{t}_{k^{\prime}}^{(v)}\right) \hat{g}_{s, k^{\prime}}\right|^{2}}
$$

where $\bar{x}$ is the conjugate of $x$, and

$$
\hat{g}_{s, k^{\prime}}=\rho_{k, k^{\prime},\left|l-l^{\prime}\right|} \hat{b}_{k^{\prime}, s-1}+\bar{\rho}_{k, k^{\prime},\left|l-l^{\prime}\right|} \hat{b}_{k^{\prime}, s}
$$

Note that $\mathbf{X}^{(i)}$ denotes the $i$ th element of the vector matrix $\mathbf{X}$ (notation is adapted with multiple indexes in cases of multidimensional matrices).

Transmission vectors are formulated according to the iterative algorithm of Algorithm 1. In Step 1 , all vector values are initialized, where $\mathbf{1}(d)$ is a $d \times 1$ matrix of ones. In Step 2, the equivalent matrix $\mathbf{A}_{k, i}$ of the desired MS signal at the $i$ th iteration is calculated, and the transmit vector is updated as the eigenvector $\mathbf{X}$ that corresponds to the maximum eigenvalue of $\mathbf{A}_{k, i}$ (i.e., $\lambda_{m}\left(\mathbf{A}_{k, i}\right)$ ). The algorithm comes to an end when the transmit power $p_{k}$ converges, according to threshold $\varepsilon$.

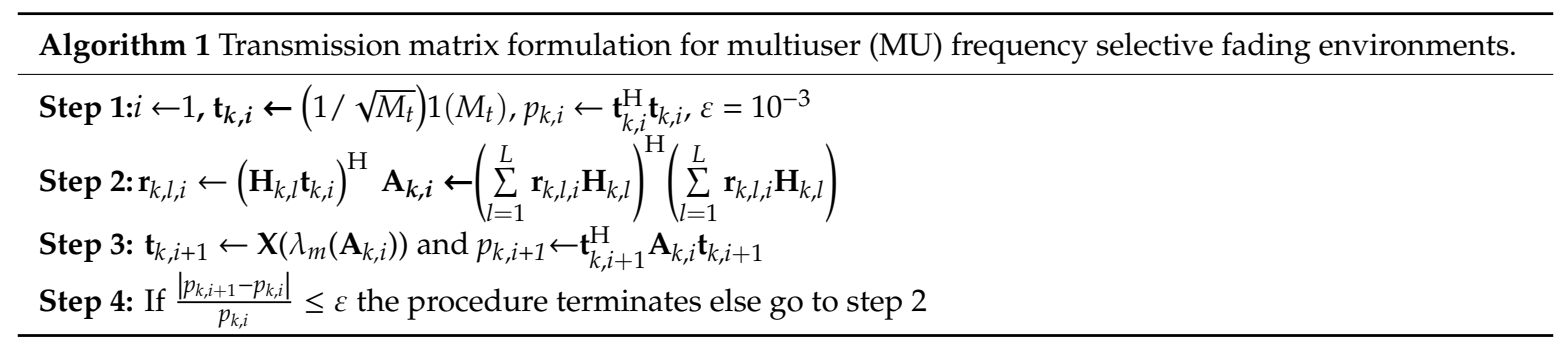




\section{Code Reuse via Principal Component Analysis}

As it was shown in [21], PCA can lead to transmission complexity reduction in MIMO-CDMA wireless configurations. In this context, only the primary eigenvector of the corresponding correlation matrix $\mathbf{C}_{k}$ of received data is sent back to the transmitter, which formulates a modified matrix $\widetilde{\mathbf{C}}_{k}$

$$
\begin{gathered}
\mathbf{C}_{k}\left(l, l^{\prime}\right)=\frac{1}{S} \sum_{s=1}^{S} \zeta_{k, l, s} \overline{\zeta_{k, l^{\prime}, s}} 1 \leq l, l^{\prime} \leq L \\
\widetilde{\mathbf{C}}_{k}=\mathbf{V}(1: L, 1: w)^{\mathrm{H}} \Sigma(1: w, 1: w) \mathbf{V}(1: L, 1: w)
\end{gathered}
$$

In Equations (5) and (6), $S$ is the total number of samples and $w$ is the number of equivalent eigenvectors (denoted as $\mathbf{V}$ ) and eigenvalues in the diagonal matrix $\Sigma$ for correlation matrix reconstruction (in our case $w$ equals 1 in order to reduce overall feedback burden [21]). Notation $a: b$ indicates all elements from $a$ to $b$ with a unity step. Note that the $(i, j)$ entry of $\mathbf{C}_{k}$ represents the overall correlation among two different reception branches in the 2-D space-time RAKE receiver. For MSs with increased channel quality, or in cases of limited multiple access interference, it is expected that non-diagonal terms of correlation matrices will be significantly reduced compared to the diagonal ones. In the proposed approach, which is described in Algorithm 2, MS grouping is performed as follows

$$
\left(u_{0}, u_{1}\right) \leftarrow \underset{\left(k, k^{\prime}\right) \in J_{U_{0}}}{\operatorname{argmax}}\left\{\frac{\prod_{l=1}^{L}\left|\widetilde{\mathbf{C}}_{k}(l, l)\right|}{\mathbf{t}_{k}^{\mathrm{H}} \mathbf{t}_{k^{\prime}} \sum_{l=1}^{L} \sum_{l^{\prime}=1, l^{\prime} \neq l}^{L}\left|\widetilde{\mathbf{C}}_{k}\left(l, l^{\prime}\right)\right|^{2}}\right\}
$$

In Equation (7), product usage ensures that weak channel conditions in certain transmission modes are properly weighted. MSs $u_{0}$ and $u_{1}$ are selected as a NOMA group (NG) (NG throughout the rest of this manuscript, while $N G_{m}$ is the maximum number of NGs) that now share the same allocated code (i.e., the one originally assigned to MS $u_{0}$ ). For a set of $K$ active MSs (denoted as $U_{0}$ ), then $J_{U_{o}}$ indicates all possible $2 \times\left(\begin{array}{c}K \\ 2\end{array}\right)=\frac{K !}{(K-2) !}$ pair combinations (note that the argument of Equation (7) is not symmetric). Afterwards, SIC is performed per NG, starting with the decoding of the symbol of user $u_{0}$ (see Algorithm 2, where $C$ denotes the transmitted symbol constellation). For example, if $K=4$ active users, then $U_{0}=\{1,2,3,4\}$ and $J_{U_{o}}=\{(1,2),(1,3),(1,4),(2,3),(2,4),(3,4),(2,1),(3,1),(4,1),(3,2),(4,2),(4,3)\}$. Considering now 2 NGs, then if for example in the first iteration the set $(2,3)$ of MSs maximizes the performance metric of Equation (7), $U_{1} \leftarrow\{2,3\}, U_{o}=\{1,4\}$ and $J_{U_{o}}=\{(1,4),(1,5),(4,5),(4,1),(5,1),(5,4)\}$. In this case, in the next step unavoidably $U_{2} \leftarrow\{1,4\}, U_{0} \leftarrow\{\}$.

It should be noted at this point that in our proposed approach, transmission vector formulation is performed locally at MSs. Hence, the required feedback is minimized, since MSs send back to their serving BS only the primary eigenvector of the correlation matrix along with the corresponding eigenvalue and the transmit vector. The BS then informs all involved MSs in an NG with the transmission vectors to be used during SIC decoding. With respect to Figure 1, and unlike other proposed approaches in literature, NOMA users can now have the same transmission power levels as in the OMA case. As it will be shown in the results section, average BER values can be maintained at practically the same levels compared to OMA transmission schemes. This is achieved since NOMA grouping takes into account not only the desired MS signal and channel conditions, but interference minimization to the other MS of the same NG as well. 


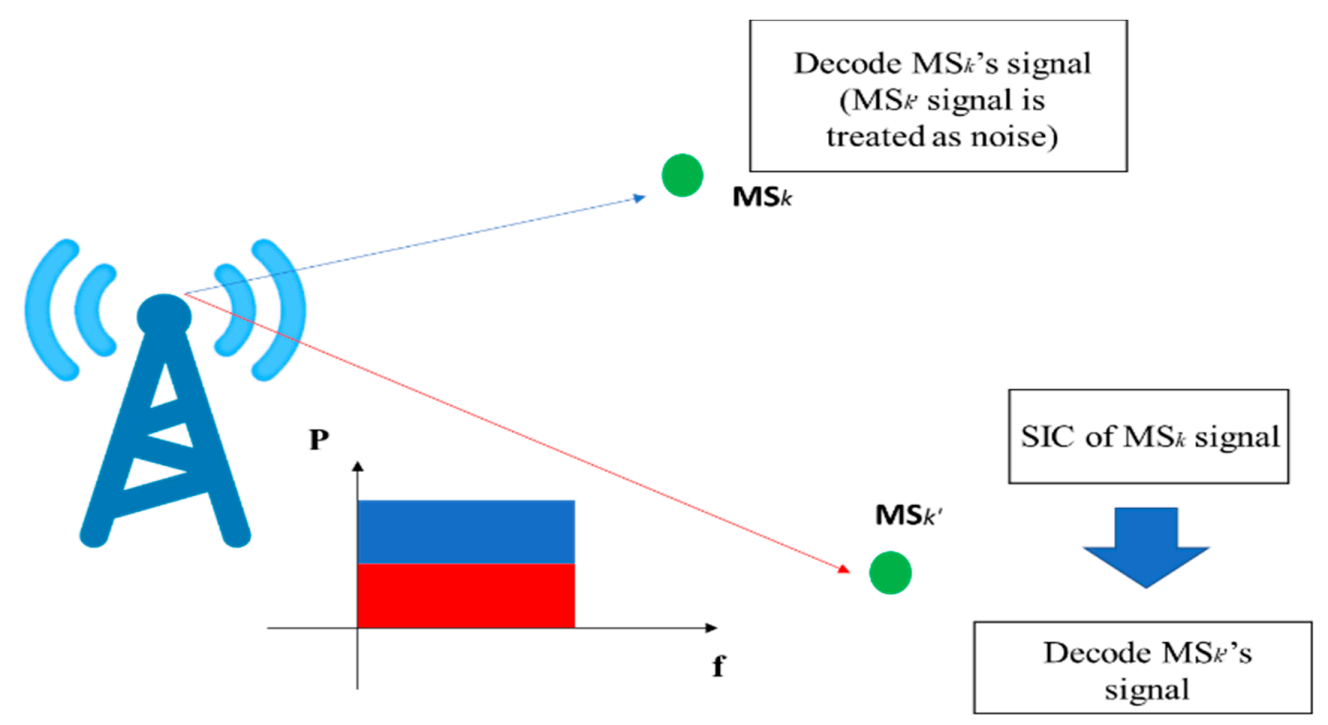

Figure 1. Successive interference cancellation (SIC) for a pair of mobile stations (MSs).

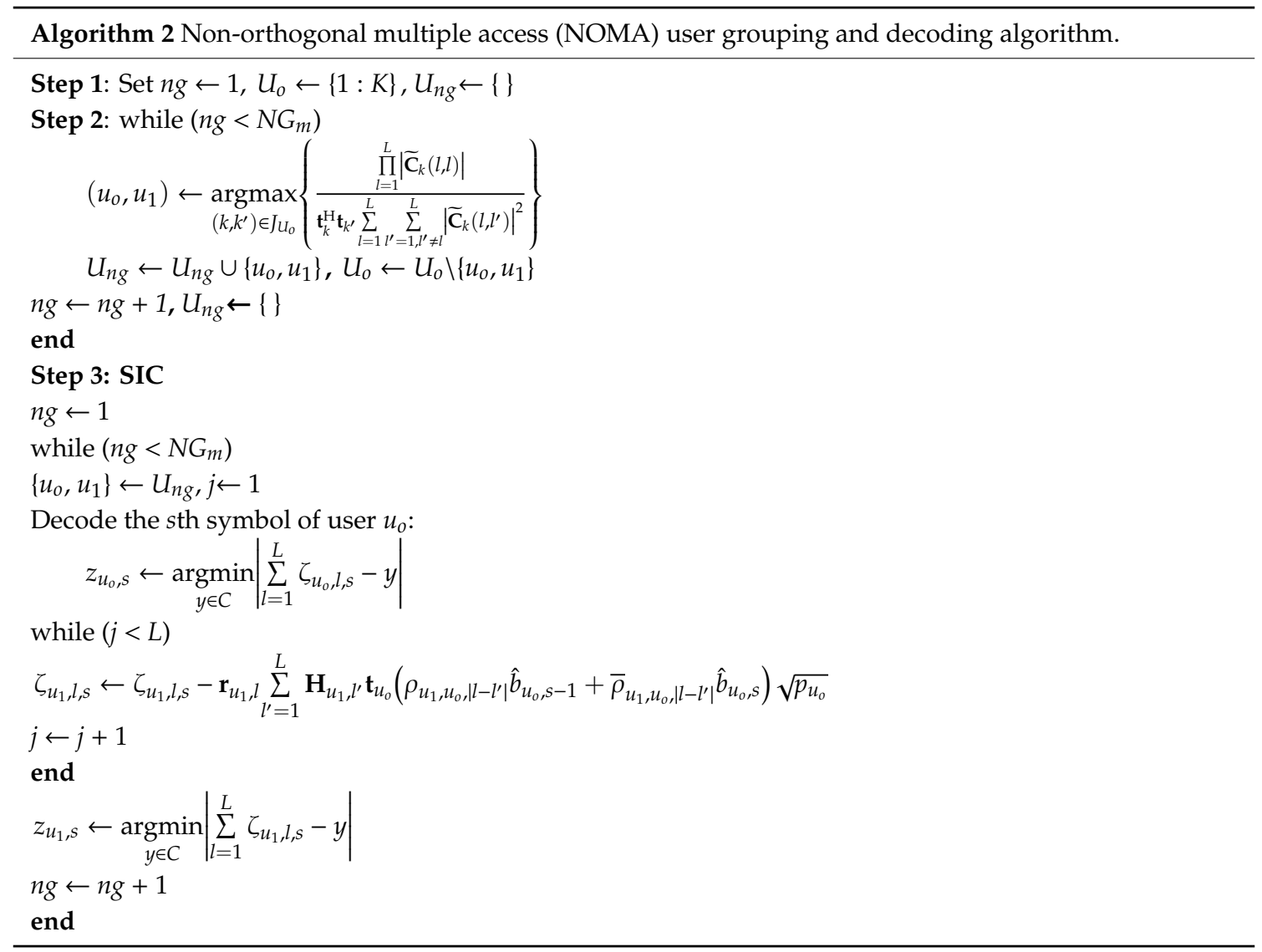

\section{Results}

The simulation results are presented in Figures 2-5 for three MIMO configurations: $4 \times 4,6 \times 4$, $8 \times 4$ with two resolvable multipath components. Effective Signal to Noise Ratio (SNR) was set to $0 \mathrm{~dB}$. The output measured metric is the average BER of all active MSs in the network, while the OMA transmission scheme was evaluated as well. Processing gain was set to 32 [23], in order to simulate increased inter-user interference scenarios. In the first set of simulation results (i.e., Figure 2), NGs can be either 2, 4, 8 or 10 while MSs may vary from 20 to 30 with a step of 2 . As can be observed, for the increased number of MSs in the network, the average BER deterioration compared to the OMA case is 
practically negligible. For 8 NGs and 30 MSs, BER in the OMA/NOMA case is $7.76 \times 10^{-4} / 7.93 \times 10^{-4}$, respectively. Hence, in this case, a CAG of almost $25 \%$ can be achieved, since 22 codes are now assigned compared to the 30 codes in the OMA scheme. For 30 active MSs in the network and 10 NGs, corresponding average BER values are now $7.76 \times 10^{-4} / 8.11 \times 10^{-4}$, respectively. Hence, in this case, BER deterioration is limited to $4.5 \%$, with an equivalent CAG that reaches $33 \%$ (20 codes in the NOMA case).

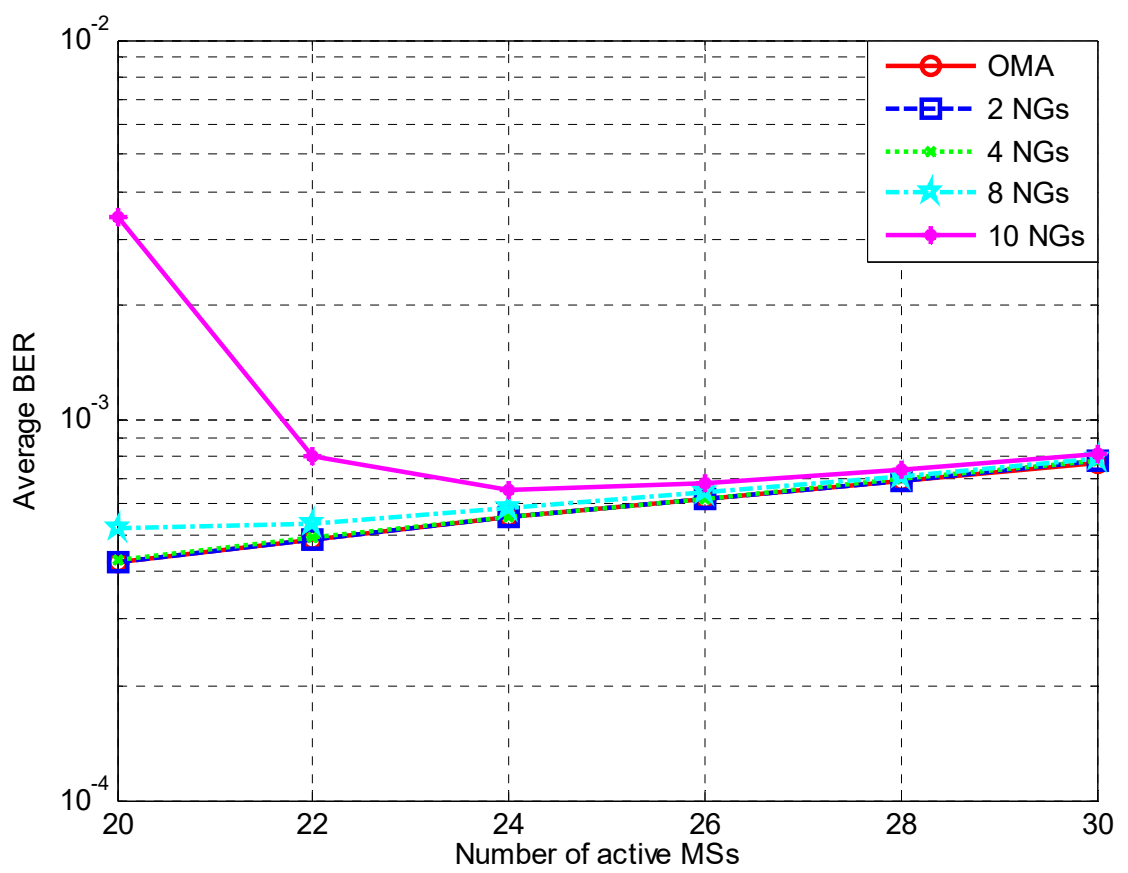

Figure 2. Bit error rate (BER) values for various NOMA groups (NGs) $-4 \times 4$ multiple-input multiple-output (MIMO) configuration.

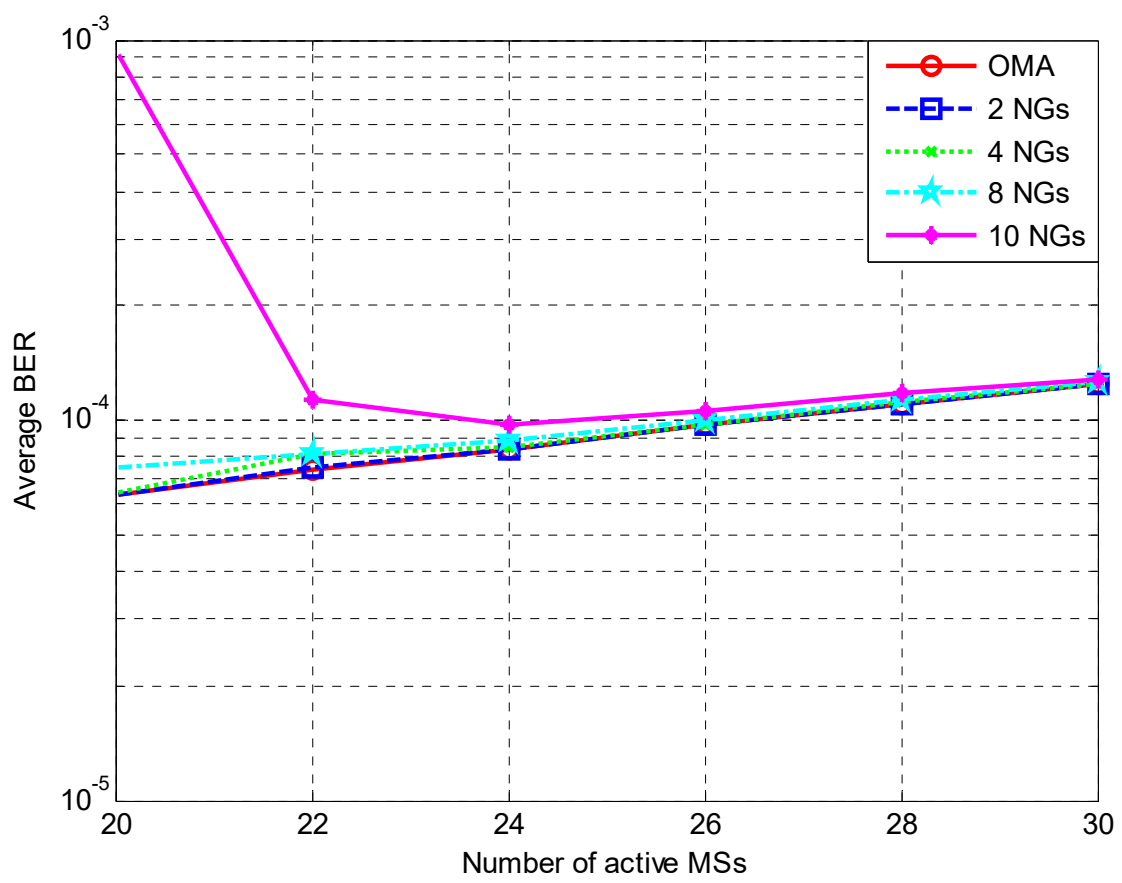

Figure 3. BER values for various NGs $-6 \times 4$ MIMO configuration. 


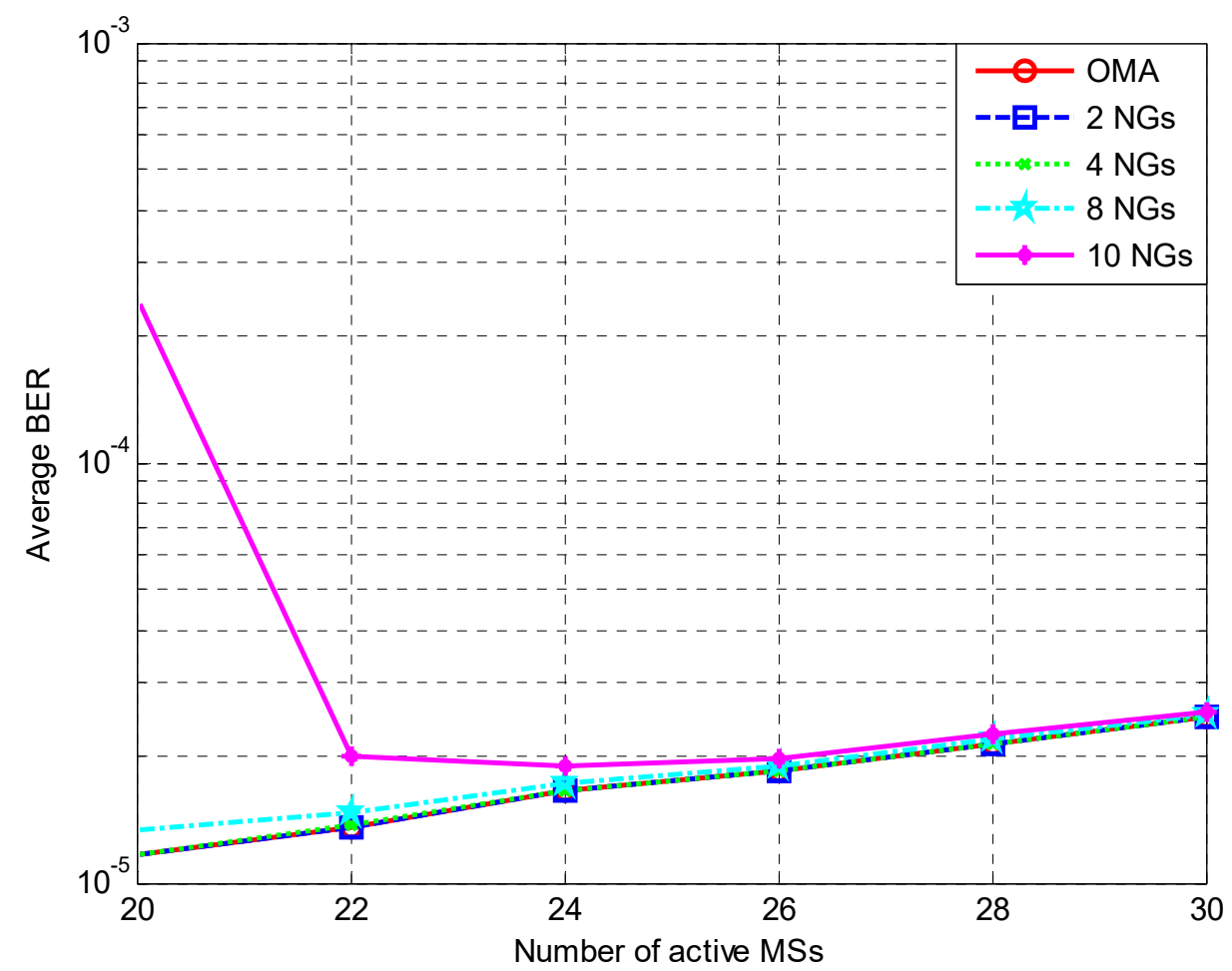

Figure 4. BER values for various NGs- $8 \times 4$ MIMO configuration.

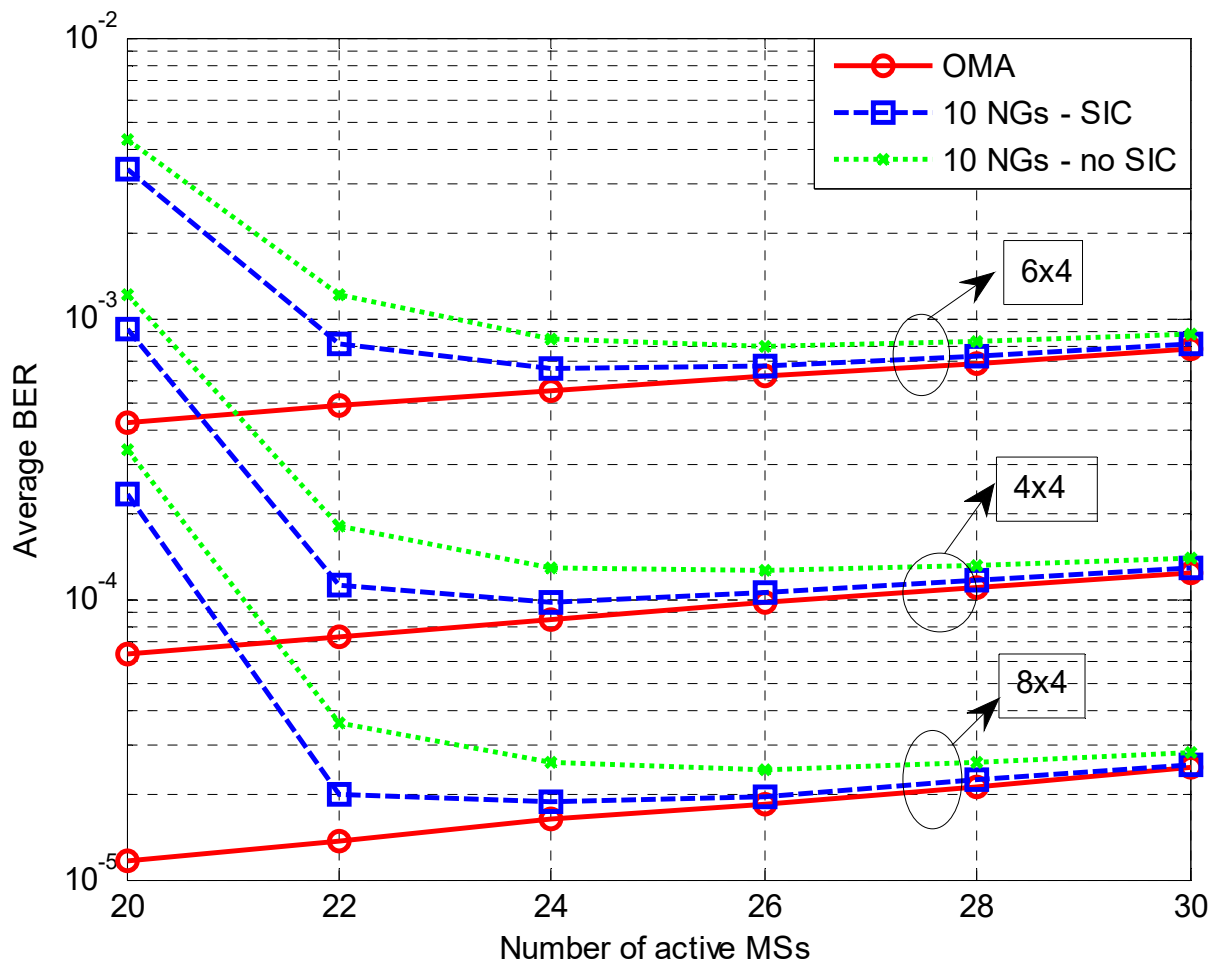

Figure 5. BER values for $10 \mathrm{NGs-Comparison} \mathrm{with} \mathrm{the} \mathrm{non-SIC} \mathrm{approach.}(4 \times 4,6 \times 4,8 \times 4 \mathrm{MIMO}$ configurations). 
In Figures 3 and 4, a $6 \times 4$ and a $8 \times 4$ MIMO configuration were considered, respectively. As it can be observed, the average BER deterioration is improved when considering 30 active users and 10 NGs, since the diversity order (i.e., $M_{t} \times M_{r}$ ) of the system is increased: The corresponding values are $1.23 \times 10^{-4} / 1.28 \times 10^{-4}$ in the $6 \times 4$ case and $2.48 \times 10^{-5} / 2.56 \times 10^{-5}$ in the $8 \times 4$ case. Hence, average BER deterioration is limited to $4 \% / 3 \%$, respectively.

In Figure 5, considering 10 NGs, SIC has been omitted in order to evaluate the potential degradation of our proposed approach compared to the OMA case. The average BER value in the NOMA scheme is now $8.8 \times 10^{-4}$ considering the $4 \times 4$ MIMO configuration. Therefore, although the BER degradation compared to OMA transmission is now almost $13 \%$, CAG values of $33 \%$ are still achieved. Hence, depending on the tolerable BER per requested service, a trade-off can be made between average BER and transceiver complexity reduction. In the $8 \times 4$ configuration, the corresponding BER values are $2.81 \times 10^{-5} / 2.53 \times 10^{-5}$ for the OMA/NOMA case, respectively. Therefore, the average BER degradation is further reduced to $11 \%$.

Unavoidably, SIC introduces a decoding delay. Hence, BER degradation can be alternately mitigated via channel encoding techniques, where the trade-off dipole is now the average BER/net bit rate [26,27]. Moreover, it should be noted that BER in the NOMA case can significantly degrade for a reduced number of MSs in the network and an increased number of NGs. This is rather expected, since in this case multiuser diversity is reduced, and the derived performance metric of Equation (7) will unavoidably select pairs with increased interuser interference. Finally, performance gain values come in agreement with the results presented in [14], where an Orthogonal Frequency Division Multiple Access (OFDMA) NOMA scheme was considered.

\section{Conclusions}

The performance of a proposed NOMA transmission scheme was evaluated for a varied number of active users and NOMA groups, in a multiuser MIMO configuration. According to the presented results, a significant code assignment gain can be obtained, with minimum BER deterioration and SIC at MSs. This is achieved with minimum computational complexity and overall feedback burden, as MSs send back to their serving BS only the primary eigenvector and the corresponding eigenvalue of the received data correlation matrix, deduced via PCA. However, even in the absence of SIC, BER degradation can be mitigated by channel encoding techniques.

Future work includes, among other things, the extension of the presented algorithm in 5G multicellular configurations, where user grouping should incorporate additional selection criteria, such as requested service, intercell interference, as well as handover rate especially in massive MIMO systems.

Author Contributions: Conceptualization, P.K.G., P.T.T. and L.E.S.; methodology, P.K.G., P.T.T. and L.E.S.; software, P.K.G.; validation, P.K.G., P.T.T. and L.E.S.; formal analysis, P.K.G., P.T.T. and L.E.S.; investigation, P.K.G.; resources, P.K.G. and P.T.T.; data curation, P.K.G. and P.T.T.; writing-original draft preparation, P.K.G.; writing-review and editing, P.K.G., P.T.T. and L.E.S.; visualization, P.K.G. and P.T.T. All authors have read and agreed to the published version of the manuscript.

Funding: This research received no external funding.

Conflicts of Interest: The authors declare no conflict of interest.

\section{References}

1. Andrews, J.G.; Buzzi, S.; Choi, W.; Hanly, S.V.; Lozano, A.; Soong, A.C.K.; Zhang, J.C. What Will 5G Be? IEEE J. Sel. Areas Commun. 2014, 32, 1065-1082. [CrossRef]

2. Gupta, A.; Jha, R.K. A Survey of 5G Network: Architecture and Emerging Technologies. IEEE Access 2015, 3, 1206-1232. [CrossRef]

3. Trakadas, P.T.; Nomikos, N.; Michailidis, E.T.; Zahariadis, T.V.; Facca, F.M.; Breitgand, D.; Rizou, S.; Masip-Bruin, X.; Gkonis, P.K. Hybrid Clouds for Data-Intensive, 5G-Enabled IoT Applications: An Overview, Key Issues and Relevant Architecture. Sensors 2019, 19, 3591. [CrossRef] [PubMed] 
4. Cheng, X.; Tang, C.; Zhang, Z. Accurate Channel Estimation for Millimeter-Wave MIMO Systems. IEEE Trans. Veh. Technol. 2019, 68, 5159-5163. [CrossRef]

5. Uwaechia, A.N.; Mahyuddin, N.M. A Comprehensive Survey on Millimeter Wave Communications for Fifth-Generation Wireless Networks: Feasibility and Challenges. IEEE Access 2020, 8, 62367-62414. [CrossRef]

6. Ji, H.; Kim, Y.; Onggosanusi, E.; Nam, Y.; Lee, J.; Zhang, J.; Lee, B.; Lee, K. Overview of Full-Dimension MIMO in LTE-Advanced Pro. IEEE Commun. Mag. 2016, 55, 176-184. [CrossRef]

7. Mokhtari, Z.; Sabbaghian, M.; Dinis, R. A Survey on Massive MIMO Systems in Presence of Channel and Hardware Impairments. Sensors 2019, 19, 164. [CrossRef]

8. Dai, L.; Wang, B.; Ding, Z.; Wang, Z.; Chen, S.; Hanzo, L. A Survey of Non-Orthogonal Multiple Access for 5G. IEEE Commun. Surv. Tutorials 2018, 20, 2294-2323. [CrossRef]

9. Ding, Z.; Lei, X.; Karagiannidis, G.K.; Schober, R.; Yuan, J.; Bhargava, V.K. A Survey on Non-Orthogonal Multiple Access for 5G Networks: Research Challenges and Future Trends. IEEE J. Sel. Areas Commun. 2017, 35, 2181-2195. [CrossRef]

10. Dai, L.; Wang, B.; Yuan, Y.; Han, S.; Chih-Lin, I.; Wang, Z. Non-orthogonal multiple access for 5G: Solutions, challenges, opportunities, and future research trends. IEEE Commun. Mag. 2015, 53, 74-81. [CrossRef]

11. Zeng, M.; Yadav, A.; Dobre, O.A.; Tsiropoulos, G.I.; Poor, H.V. Capacity Comparison Between MIMO-NOMA and MIMO-OMA With Multiple Users in a Cluster. IEEE J. Sel. Areas Commun. 2017, 35, 2413-2424. [CrossRef]

12. Hoshyar, R.; Wathan, F.P.; Tafazolli, R. Novel Low-Density Signature for Synchronous CDMA Systems Over AWGN Channel. IEEE Trans. Signal Process. 2008, 56, 1616-1626. [CrossRef]

13. Ding, Z.; Fan, P.; Poor, H.V. Impact of User Pairing on 5G Nonorthogonal Multiple-Access Downlink Transmissions. IEEE Trans. Veh. Technol. 2016, 65, 6010-6023. [CrossRef]

14. Saito, Y.; Kishiyama, Y.; Benjebbour, A.; Nakamura, T.; Li, A.; Higuchi, K. Non-Orthogonal Multiple Access (NOMA) for Cellular Future Radio Access. In Proceedings of the 2013 IEEE 77th Vehicular Technology Conference (VTC Spring), Dresden, Germany, 2-5 June 2013; pp. 1-5.

15. Ding, Z.; Adachi, F.; Poor, H.V. The Application of MIMO to Non-Orthogonal Multiple Access. IEEE Trans. Wirel. Commun. 2015, 15, 537-552. [CrossRef]

16. Nomikos, N.; Trakadas, P.T.; Hatziefremidis, A.; Voliotis, S. Full-Duplex NOMA Transmission with Single-Antenna Buffer-Aided Relays. Electronics 2019, 8, 1482. [CrossRef]

17. Nomikos, N.; Michailidis, E.T.; Trakadas, P.; Vouyioukas, D.; Zahariadis, T.; Krikidis, I. Flex-NOMA: Exploiting Buffer-Aided Relay Selection for Massive Connectivity in the 5G Uplink. IEEE Access 2019, 7, 88743-88755. [CrossRef]

18. Al-Abbasi, Z.Q.; So, D.K.C. User-Pairing Based Non-Orthogonal Multiple Access (NOMA) System. In Proceedings of the 2016 IEEE 83rd Vehicular Technology Conference (VTC Spring), Nanjing, China, 15-18 May 2016; pp. 1-5.

19. Zhu, L.; Zhang, J.; Xiao, Z.; Cao, X.; Wu, D.O. Optimal User Pairing for Downlink Non-Orthogonal Multiple Access (NOMA). IEEE Wirel. Commun. Lett. 2019, 8, 328-331. [CrossRef]

20. Liu, F.; Mähönen, P.; Petrova, M. Proportional fairness-based user pairing and power allocation for non-orthogonal multiple access. In Proceedings of the 2015 IEEE 26th Annual International Symposium on Personal, Indoor, and Mobile Radio Communications (PIMRC), Hong Kong, China, 30 August-2 September 2015; pp. 1127-1131.

21. Gkonis, P.K.; Kaklamani, D.-T.I.; Venieris, I.S.; Dervos, C.T.; Chryssomallis, M.; Siakavara, K.; Kyriakou, G.A. On the Reduction of Transmission Complexity in MIMO-WCDMA Frequency-Selective Fading Orientations via Eigenvalue Analysis. Electronics 2018, 7, 239. [CrossRef]

22. Gkonis, P.K.; Tsoulos, G.; Kaklamani, D. Dual code Tx diversity with antenna selection for spatial multiplexing in MIMO-WCDMA networks. IEEE Commun. Lett. 2009, 13, 570-572. [CrossRef]

23. Holma, H.; Toskala, A. (Eds.) WCDMA for UMTS. Radio Access for Third Generation Mobile Communications, 3rd ed.; Wiley: Hoboken, NJ, USA, 2004.

24. Cai, Y.; Qin, Z.; Cui, F.; Li, G.Y.; McCann, J.A. Modulation and Multiple Access for 5G Networks. IEEE Commun. Surv. Tutor. 2018, 20, 629-646. [CrossRef] 
25. Gkonis, P.K.; Kaklamani, D.-T.I. Reduced Complexity BER Calculations in Large Scale Spatial Multiplexing Multi-User MIMO Orientations in Frequency Selective Fading Environments. Electronics 2019, 8, 727. [CrossRef]

26. Chen, P.; Xie, Z.; Fang, Y.; Chen, Z.; Mumtaz, S.; Rodrigues, J.J.P.C. Physical-Layer Network Coding: An Efficient Technique for Wireless Communications. IEEE Netw. 2020, 34, 270-276. [CrossRef]

27. Battaglioni, M.; Tasdighi, A.; Cancellieri, G.; Chiaraluce, F.; Baldi, M. Design and Analysis of Time-Invariant SC-LDPC Convolutional Codes with Small Constraint Length. IEEE Trans. Commun. 2017, 66, 918-931. [CrossRef]

(C) 2020 by the authors. Licensee MDPI, Basel, Switzerland. This article is an open access article distributed under the terms and conditions of the Creative Commons Attribution (CC BY) license (http://creativecommons.org/licenses/by/4.0/). 